\title{
Anticoagulant Utilization Evaluation in a Tertiary Care Teaching Hospital: An Observational Prospective Study in Medical in Patients
}

\author{
Vijay Singh, Krishnappa Gopinath, Amirali Behzadpour and Neelathahalli Kasturiranagan Meera* \\ Department of Pharmacy Practice, Visveswarapura Institute of Pharmaceutical Sciences, $24^{\text {th }}$ main, $22^{\text {nd }}$ cross Bana shankari, \\ $2^{\text {nd }}$ Stage, Bangalore, India.
}

\begin{abstract}
Introduction: Drug utilization evaluation of anticoagulant drugs is essential considering the spectrum of use and associated risks with their therapy. Objectives: The objectives of the present study were to evaluate the drug utilization pattern of anticoagulants, identify and assess drug interactions and ADRs with the use of anticoagulants. Method: 84 patients from the medicine department were included in this prospective, cross sectional study conducted for one year. Results: Heparin was mainly used for prophylaxis. Enoxaparin and acenocoumarol were other commonly used drugs. The diagnosis varied from Ischemic heart disease (IHD), deepvein thrombosis (DVT), pulmonary embolism (PE), cardiovascular accident and others. Laboratory tests like prothrombin time (PT), international normalized ratio (INR), activated partial thromboplastin time (aPTT), in few cases radiological tests were performed to monitor the therapy. Use of mono therapy and combination therapy aswell as topical use of heparingel was observed. Acenocoumarol and warfarin were the drugs prescribed at discharge. 35 drug interactions were identified, twelve pharmacokinetic and twenty three pharmaco dynamic in nature. Acenocoumarol was the most commonly identified drug with drug interactions. Pharmacokinetic mechanism commonly found was inhibition of vitamin Kactivity; synergistic effect was the mechanism of pharmaco dynamic interaction. Sixteen interactions were severe in intensity. 6 adverse drug reactions (ADRs) were observed during the study. Conclusion: The pharmacotherapy with anticoagulant drugs should be cost effective and with minimum risks involved. The study highlights the importance of following the guidelines for appropriate use of anticoagulants.
\end{abstract}

Key words: Anticoagulant, Drug utilization pattern Prothrombin time, Drug interactions, International normalized ratio.

\section{INTRODUCTION}

The primary intent of drug utilization research is to smooth the process \& rational use of drugs in population. Drug Utilization Evaluation (DUE) is a study to identify variability in drug use \& to support interventions that will improve patient's therapeutic outcomes. Drug use indicators are intended to measure specific aspects of health providers \& drug use in a hospital or health center. Indicators will provide information to health care managers concerning drug use, prescribing habits \& important views of patient care. ${ }^{1}$

Anticoagulants are the drugs used to prevent thrombus extension and embolic complications by reducing the rate of fiber in forma- tion. They do not dissolve already formed clot but prevent recurrences. Anticoagulants have the ability to prevent devastating medical complications. In fact, venous thromboembolism (VTE) is considered the most preventable cause of hospital death in the United States.

In a hospital setting anticoagulants are mainly used for the following indications like deepveinthrombosis (DVT), pulmonary embolism (PE), myocardial infarction (MI), unstable angina, rheumatic heart disease, vascular surgery, prosthetic heart valve, retinal vessel thrombosis, extra corpuscular circulation, hemodialysis \& defibrination syndrome. Without prophylaxis, the inci-
Submitted date : 26/02/2015 Accepted date :05/06/2015

DOI: 10.5530/ijopp.8.2.3

Address for correspondence: Dr. Neelathahalli Kasturiranagan Meera, Department of Pharmacy Practice, Visveswarapura Institute of Pharmaceutical Sciences, $24^{\text {th }}$ main, $22^{\text {nd }}$ cross Bana shankari, $2^{\text {nd }}$ Stage, Bangalore-560070, India. E-mail:meera_satish@yahoo. com

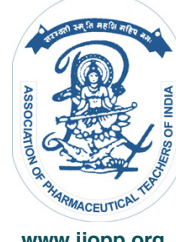


dence of hospital-acquired deepveinthrombosis (DVT) in general medical and surgical patients range from 10 to $40 \%$. The incidence increases to roughly $50 \%$ in patients with no prophylaxis prior to major orthopedic surgery. ${ }^{2}$

Prophylactic anticoagulants have been shown to significantly reduce this risk. Therapeutic anticoagulation is the cornerstone of treatment for thromboembolic disease. However, titrating anticoagulant dose with in narrow therapeutic indices at times proves difficult, even for the most experienced practitioners. Individual patient response to a standard dose is not always predictable. Variation in patient-specific factors such as rate of drug elimination further complicates the picture. Overanticoagulation exposes patients to increased risk of hemorrhage; one of the most feared hospital complications. Under-anticoagulation leaves patients vulnerable to recurrent VTE consequently, heparin, warfarin, and enoxaparin are ranked in the United States Pharmacopoeia (USP) as Top 50 Drug Products Associated with Medication Errors at number 5, 7, and 12, respectively. ${ }^{3}$

Due to the importance of anticoagulant use along with the complications that VTE\&PT may bring about for patients this study on utilization pattern of anticoagulants prescribed routinely along with the study of drug inter actions and adverse drug reaction was performed.

\section{METHODOLOGY}

This prospective cross sectional study was conducted in a tertiary care teaching hospital in Bengaluru city, providing specialized health care services to all strata of people in and around Bangalore, having more than 1000 beds in various specialty wards. The study included a total of 84 patients receiving anticoagulant drug therapy from Medicine ward, with a variety of underlying conditions who were admitted during the study period of 1year. Patients of either gender and more than 18 years of age, were included in the study. Patients on hemodialysis and therefore receiving anticoagulant treatment were excluded from the studies. For every patient, data was recorded in a pre-designed form which included data like demographics such as age, gender, habitat, occupation, education, income, underlying disease, indication of anticoagulant use and various diagnostic data regarding diagnosis and anticoagulant therapy monitoring (PT, INR, aPTT) before and till the time of discharge. Source of data included patient case records, treatment charts and laboratory reports. During the study patient's medication chart was also monitored for any drug-drug interaction involving the anticoagulants used and any adverse drug reactions experienced by the patients on anticoagulant treatment.
The study was approved by the Human ethical clearance committee of Visveswarapura Institute of Pharmaceutical Sciences (VIPS), Bengaluru.

\section{Statistical Analysis}

The data was analyzed using Microsoft excel and SPSS version 16.0. The results are expressed in terms of number and percentage. The drug-drug interactions were analyzed for severity using American Hospital Formulary System (AHFS) Drug Hand Book, Stockley's drug interactions, $9^{\text {th }}$ Edition \& www.drugs.com as the resource. ADRs observed were assessed for causality using Naranjo's causality scale.

\section{RESULTS}

During the study, 84 patients were administered anticoagulants, $46(69 \%)$ of them were males and $38(31 \%)$ were females. These patients were categorized into age groups which showed, 19(23\%) were belonging to age group of 61 to 70 years which covered majority of patients in the study, followed by $15(18 \%)$ patients in age group of 31 to 40 years, patients in age groups of each 41 to 50,51 to 60 and 71 to 80 were found to be same in distribution which was $11(13 \%) ; 10(12 \%)$ patients belonged to more than 80 years age group. Age group of 61 to 70 years was the most common in both gender (Figure1).

Out of 84 patients, 53(63\%) were from urban region and $31(37 \%)$ from rural region. Education status showed $28(39 \%)$ of patients had no formal education where as patients with educational status from primary to graduation showed a close distribution pattern.

Occupation details of study patients revealed that out of 38 female patients $29(35 \%)$ were housewife, $30(36 \%)$ patients were self-employed, 18(21\%) patients reported to be employed and $7(8 \%)$ were having other types of occupation. In this study self-declared annual family income was documented, $9(12 \%)$ had income less than 1 lakh rupees, 48(63\%) patients reported 1-3 lakh rupees, $17(22 \%) 3-5$ lakh rupees and only $3(3 \%)$ patients of more than 5 lakh rupees. 7 patients did not report of having income source and their therapy was funded by non-government organizations (NGOs). Table 1 depicts the various reasons for admission of the patients included in the study to the hospital.

Among the study patients, 48(57\%) patient's laboratory tests were evaluated for parameters like PT, INR and aPTT. In the remaining 36 patients the above tests were not performed, how ever in many of these patients radiological diagnostic evaluations like Magnetic resonance imaging (MRI), angiography, computer- 


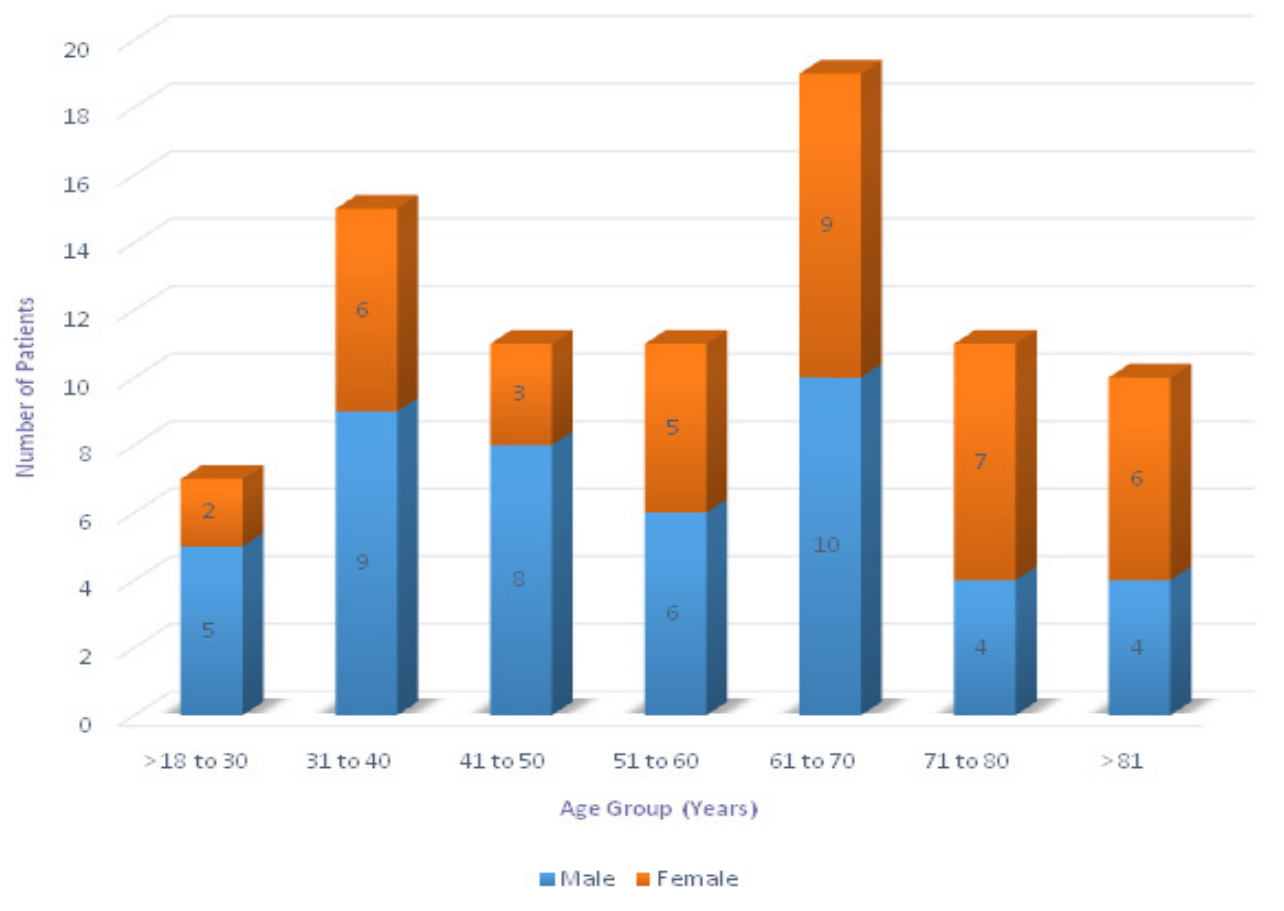

Figure 1: Distribution of patients based on gender and age

\section{Table 1: Reasons for Hospital Admission}

\begin{tabular}{lcc}
\multicolumn{1}{c}{ Cardiovascular Conditions } & \multicolumn{2}{c}{ Non Cardiovascular Conditions } \\
\hline Congenital Heart Diseases-1 & Hematological & Non Hematological \\
Ischemic Heart Disease-13 & Partial Thromboembolism-2 & Cerebrovascular accident-6 \\
Cardiovascular Accident-3 & Microcyticanemia-1 & COPD-2 \\
Rheumatic Heart Disease-3 & Dengue with & Bronchitis-1 \\
Cor Pulmonale-2 & Thrombocytopenia-1 & Respiratory failure due to Left \\
Coronary Angioplasty Stenting-1 & Peripheral artery disease-1 & Lung failure-1 \\
Myocardial Infarction-4 & Iron deficiency anemia-1 & Bronchial asthma with Diabetes \\
Hyper Obstructive Cardiomyopathy- & PE-4 & Mellitus-2 \\
1 & DVT-9 & Esophageal Varices-1 \\
R.VSoftClot-2 & VT-2 & Diabetic Foot-1 \\
Dilated Cardiomyopathy-1 & PE+DVT-5 & Lower limb cellulitis-1 \\
L.Vapicalclot-1 & Cerebral Veinthrombosis-1 & Pulmonary Stenosis-1 \\
RHD with Cardiovascular accident & Portal HTN with & Extra hepaticportalvein \\
Hemiparesis-5 & coagulopathy-1 & obstruction-1 \\
IHD+Cerebrovascular accident-2 & - & Fracture-2 \\
\hline
\end{tabular}

Note: Ischemic heart disease(IHD); Rheumatic heart disease (RHD); Right ventricular(RV); Left ventricular(LV); Pulmonary embolism (PE); Deep vein thrombosis (DVT); Venous thrombosis (VT); Hypertension (HTN); Chronic obstructive pulmonary disease (COPD).

ized tomography (CT) scan were carried out inorder to detect the presence of clots/throm bus formation.

Out of the 48 patients in whom coagulation related tests were performed, these tests were repeated in 36 patients during their stay in the hospital in order to monitor the efficacy of the anticoagulant therapy. Incase of 12 patients the findings with PT, INR, aPTT tests were found to show increase where as in 24 other patients the elevated values decreased close to the normal values with the anticoagulant therapy. In remaining 12 patients, the tests were performed only once during the hospital stay and the test values were found to be close to the normal values. In addition to these lab tests few patients were also evaluated with radiological findings.

From 84 patients, anti-coagulant us age was seen as total of 97 cases including change in drug used during the 


\begin{tabular}{lcc}
\hline \multicolumn{3}{|c|}{ Table 2: Categories of anticoagulant drugs used } \\
\hline Class & Number & Percentage (\%) \\
\hline $\begin{array}{l}\text { Heparin \& Derivatives } \\
\text { Heparin }\end{array}$ & 24 & 25 \\
Low Molecular Weight Heparin (LMWH) & \\
• Enoxaparin & 30 & 31 \\
• Lomorin & 1 & 1 \\
Coumarin Derivatives & & \\
• Acenocoumarol & 37 & 38 \\
- Warfarin & 5 & 5 \\
Total & 97 & 100 \\
\hline
\end{tabular}

hospital stay. Of these 50(52\%) cases were administered with parenteral drugs, in $41(43 \%)$ cases received oral administration and $6(6 \%)$ cases used topical heparin gel. Table 2 shows the categories of drugs used in the study.

$63(84 \%)$ patients received only one anticoagulant drug during hospitalization. However in 21(16\%) patients more than one anticoagulant drug was used. This combination therapy included parenteral and oral drugs, or combination of two or more drugs administered orally. Most common combination therapy was acenocoumarol+enoxaparin(4), acenocoumarol+heparin(3) followed by enoxaparin $+h$ eparin+warfarin, enoxaparin + acenocoumarol+heparin, enoxaparin+warfarin, enoxaparin +heparin was used in the hospital.

At the time of discharge, 40 patients were prescribed with anticoagulants; $7(18 \%)$ patients were prescribed with warfarin and the rest $33(82 \%)$ patients were given acenocoumarol, both by oral route of administration. 26 patient who were given acenocoumarol and all patients on warfarin had been instructed to continue the therapy every day.while the remaining 7 patients on acenocoumarol were instructed to take the drug ranging from 2 to 4 weeks and then review the therapy after repeat laboratory tests.

Concurrent use of antiplatelet aggregating drugs was also observed during the study. The patients were prescribed with aspirin alone, aspirin+clopidogrel, and clopidogrel alone. Similar such treatment was also reported in another study. ${ }^{4}$

As another objective of study, the patient's medication chart was evaluated for drug interactions involving anticoagulants. A total of 35 drug interactions were identified. In 24 of interaction identified Acenocoumarol was involved in interaction with other drugs like a moxicillin, cephalosporin's, enoxaparin, pentoxyfylline. 9 interaction were observed involving heparin with drugs like tirofiban, aspirin, clopidogrel and piperacillin. Enoxopa- rin with pentoxyfylline and warfarin with phenytoin were the other interactions identified.

Out of 35 interactions 12 were pharmacokinetic by nature and the rest 23 were pharmacodynamic interactions. The pharmacokinetic drug interaction between acenocoumarol \& amiodarone is due to the mechanism that acenocoumarolacts by inhibiting Vitamin K synthesis and amiodarone acts by inhibiting the action of cytochrome P450 isozyme, both of this action results in increased anticoagulant activity. Interaction between heparin \& piperacillin results in increased anticoagulant activity with the mechanism involved being inhibition of platelet aggregation by piperacillin. Acenocoumarol \& pentoxifylline interaction shows increased anticoagulant activity as both of them act by inhibiting the synthesis of Vitamin K. One more interaction was observed with warfarin with phenytoin where addition of phenytoin to warfarin therapy initially increased prothrombintime, but there after reduced anticoagulant effects. The mechanism of pharmacodynamic interaction was found to be a synergistic activity involving drugs like Acenocoumarol with clarithromycin, enoxaparin, pentoxifylline, amoxicillin \& ceftriaxone. Heparininte raction was seen with drugs like tirofiban, aspirin and clopidogrel. The severities of interactions were assessed using www. drugs.com. It was found that 16 were severe, 10 were moderate and 9 mild.

Total of 6 ADR's were found of which three were with heparin, two were with warfarin and one with enoxaparin. Naranjo scale was used to assess the causality and the result was one definite and two probable ADRs with heparin, both the ADRs with warfarin were probable and a definite ADR with enoxaparin. ADRs caused hematoma, bleeding, decreased hematocrit and thrombocytopenia more with heparin.

\section{DISCUSSION}

The current study showed that the anticoagulants are very commonly used for treatment and for prophylaxis. The pattern of use was based more on clinician's judgment and experience, and in few situations the usage pattern deviated from American College of Chest Physicians (ACCP) guidelines based on patient's requirements.

Over one year of study on patients ranging from age 18 to 80 years, it was observed that there were $10(11.6 \%)$ of wrong prescription of anticoagulants where patients were prescribed anticoagulant therapy without any indication. This brings in an added cost burden to the patient including cost of monitoring along with unwanted pain and longer duration of hospital stay. Being a teaching hospital, concentration of patients from lower class 
and lower middleclass is seen more and adding economic burden of this type will not be wise. Parenteral and oral types of dosage forms were preferred, Low Molecular Weight Heparin (LMWH) was the maximum preferred parenteral anticoagulant and acenocoumarol was more preferred orally followed by un fractionated heparin (UFH) \& warfarin. LMWH being the costliest of all increases the burden when cost of monitoring is added to it and when prescribing LMWH, dose adjustment has to be done in patients with renal impairment which was only seen in $45 \%$ of cases of total LMWH indicated cases. ACCP guidelines suggests that patients who have creatinine clearance $\mathrm{ClCr}<30 \mathrm{ml} / \mathrm{min}$ should receive LMWH after dosage adjustment, dose commonly administered was found to be $40 \mathrm{mg}$ /day. ${ }^{5}$ Some studies suggested increase in potassium levels when treated with LMWH which was not seen in our studies. ${ }^{6}$ LMWH accounted for highest cost incase of anticoagulants. It was used in patients with Ischemic Heart Disease (IHD), cardiovascular accident, hyper obstructive cardiomyopathy, femur fracture, cerebrovascular accident, Peripheral Artery Disease, PE, DVT, PE-DVT.

Gensparin Sodium (Lomorin) another LMWH derivative was used in one patient diagnosed with PE and DVT both, however very few drug interaction were seen involving LMWH.

UFH was the second most used parenteral in our studies where the initial dose was $5000 \mathrm{IU}$ whereas ACCP guidelines suggest 5000IU initially followed by 1000 units/hour. Monitoring was done every 2-3 days In $60 \%$ of cases where UFH was used as prophylaxis, which was not necessary. Considering patient's condition as well as initial baseline values of PT, INR \& aPTT, decision can be taken to monitor these values on a daily bases or not, as this helps in reducing the cost of repeat tests performed. ${ }^{7}$ In our study we found that UFH was preferred in 24 cases and in many cases aPTT was not performed as per the laid down guidelines and this can result in administering an in appropriate dosage to the patient resulting in bleeding and thrombosis. We also found that in all the patients who received UFH as a therapeutic dose the test value did not reach the desired aPTT levels necessitating repeate PTT tests to be performed. It's very important to check the aPTT levels to decide the dose of UFH to be given as in adequate dose can lead to patient developing DVT. ${ }^{5}$ Heparin was most commonly used in conditions like MI, IHD, coronary angioplasty stenting, DVT, chronic obstructive pulmonary disease(COPD), asa prophylactic therapy. 9 cases were found to have a drug interaction with heparin which was second highest after acenocoumarol.

Use of oral anticoagulants was more as discharge medications where as in the hospital wards maximum used oral anticoagulant was acenocaumarol followed by warfarin which is same on discharge also. Warfarin was used only for prophylaxis and closer monitoring of patients was recommended by the clinical pharmacist. Bleeding was them ostprevalent adverse effect. It was observed that in many cases acenocoumarol was given along with a parenteral anticoagulant and the monitoring was done one very second day of therapy to avoid risk of bleeding with high dose of parenteral anticoagulant therapy and also to lessen the monitoring and laboratory test cost to the patients. Such repeat, close monitoring of therapy probably could be the reason for fewer ADRs and less evere drug interactions observed compared to any other studies done previously with similar sample size. Acenocoumarol was prescribed more at the time of discharge at a dose of $2-4 \mathrm{mg} /$ day. According to ACCP guidelines the initial therapy with acenocoumarol is $8-12 \mathrm{mg} /$ day. ${ }^{5}$ Acenocoumarol was used in patient with Rheumatic Heart Disease, Corpulmonale with increased heart rate (HR), Cardiovascular accident, Coronary Angioplasty Stenting, Partial Thromboembolism, RV Soft Clot, Dilated Cardiomyopathy, Esophageal varies, PE, DVT, VT, PE-DVT, Diabetic Foot and rheumatic heart disease (RHD) with Cardiovascular accident with Hemiparesis etc. Warfarin was used in 5 patients diagnosed with IHD/Cerebrovascular Accident, DVT, and LVA pical Clot. Physicians preferred acenocoumarol more as a discharge medication because it required less monitoring compared to warfarin.

We also observed that in 48 patient's cases where PT, INR \& tests was performed in 12 patients PT, INR $\&$ aPTT levels were increasing despite anticoagulant therapy, however in such patients the dose of anticoagulant drug administered was not further altered. Also in the therapy enoxaparin showed lesser efficacy than with acenocoumarol as the PT, INR and aPTT values with enoxaparin therapy showed increase compared to normal values.

\section{CONCLUSION}

Anticoagulant drug utilization pattern follows very commonly ACCP guidelines. Appropriate use of anticoagulant therapy and any deviation from the guidelines to a large extent also depends on patient characteristics and concomitant therapy patient is receiving. Repeat monitoring of the parameters helps to evaluate the safety of anticoagulant drug use. The therapy with these drugs needs to be cost effective and reduce the complications associated with their use. However it seems prudent to choose the anticoagulant drug therapy on a patient specific basis. The need for dosage adjustments in different diagnostic situations or specific populations is very crucial. 


\section{ACKNOWLEDGEMENT}

The authors would like to acknowledge the patients and clinicians of KIMS hospital \& research center and Prin-

\section{REFERENCES}

1. Jackson MR, Danby CA, Alving BM. Heparinoid anticoagulation and topical fibrin sealant in heparin-induced thrombocytopenia. The Annals of Thoracic Surgery 1997; 64(6): 1815-7.

2. Geerts WH, Pineo GF, Heit JA. Prevention of venous thrombo embolism: the seventh ACCP conference on antithrombotic and thrombolytic therapy. Chest 2004; 126(3): 338S-400.

3. United States Pharmacopeia Top 50 Drug Products Associated with Medication Errors. Accessedat<www.usp.org/hqi/patientSafety/resources/ top50DrugErrors.html>17Apr2008. cipal \& Staff of department of Pharmacy Practice of VIPS, Bengaluru.

4. Fahimi F, Baniasadi S, Behzadnia N, et al. Enoxaparin Utilization Evaluation: An Observational Prospective Study in Medical Inpatients. Irianian Journal of Pharmaceutical Research 2008; 7(1): 77.

5. Geerts WH, Bergqvist D, Pineo GF, et al. Antithrombotic and Thrombolytic Therapy: American College of Chest Physicians Evidence-Based Clinical Practice Guidelines $8^{\text {th }}$ Edition. Chest 2008; 133 (6 suppl): 381S-453.

6. Koren-Michowitz M, Avni B, Michowitz Y, et al. Early onset of hyperkalemia in patients treated with low molecular weight heparin: a prospective study. Pharmacoepidemiol. Drug Saf. 2003; 13(5): 299-302.

7. Khalili $\mathrm{H}$, Khavidaki $\mathrm{S}$, HosseinTalasaz $\mathrm{AH}$, et al. Anticoagulant Utilization Evaluation in a Teaching Hospital: A Prospective Study. Journal of Pharmacy Practice 2010; 23(6): 579-84. 